\title{
Endoscopic ultrasound-guided gastroenterostomy for palliative drainage of an obstructed hepatico- jejunostomy loop
}

A 63-year-old man was admitted with obstructive jaundice. Computed tomography (CT) scan showed a mass in the head of the pancreas. Endoscopic retrograde cholangiopancreatography (ERCP) revealed a common bile duct stricture, which was stented, and biliary brushings confirmed primary pancreatic adenocarcinoma. As the tumor was unresectable, he underwent a double bypass operation (i.e. hepaticojejunostomy and gastrojejunostomy). The patient presented 2 months later with jaundice (bilirubin $300 \mathrm{mg} / \mathrm{dL}$ ). CT scan confirmed obstruction and dilatation of the hepaticojejunostomy loop due to tumor invasion ( $\bullet$ Fig. 1 ).

This was resulting in backflow obstruction of the bile duct, even though the metal stent remained patent. The patient was unfit for further surgery and percutaneous drainage was considered unsafe due to postsurgical anatomy.

The dilated hepaticojejunostomy loop $(\bullet$ Fig. 2) was identified at endoscopic ultrasound (EUS) using a Pentax linear echo-endoscope (Pentax, Tokyo, Japan) and accessed using a Cook cystotome (Wilson Cook, Winston-Salem, North Carolina, USA), followed by ring diathermy.

A 10-cm 4-Fr metal stent ( $\bullet$ Figs. 3 and 4) was placed without any complications, and 2 days later the bilirubin had dropped to $100 \mathrm{mg} / \mathrm{dL}$.

EUS is widely used in the drainage of pancreatic pseudocysts [1]. Piraka et al. have demonstrated that it is technically feasible to drain virtually any fluid collection as long as it is adjacent to the gastrointestinal lumen and within reach of the echoendoscope [2]. EUS has been used to drain postoperative and peripancreatic fluid collections $[3,4]$. EUS-guided drainage can offer all the benefits of radiologically guided percutaneous drainage with the additional advantage of avoidance of transcutaneous infection [5]. A short distance $(<2 \mathrm{~cm})$ between the fluid collection and viscus, lack of ascites, and maturity of the fluid cavity all decrease risk of leakage at the puncture site [2].

Our case demonstrates the use of EUS in relieving biliary obstruction by drainage of an obstructed hepaticojejunostomy loop with transgastric gastroenterostomy.

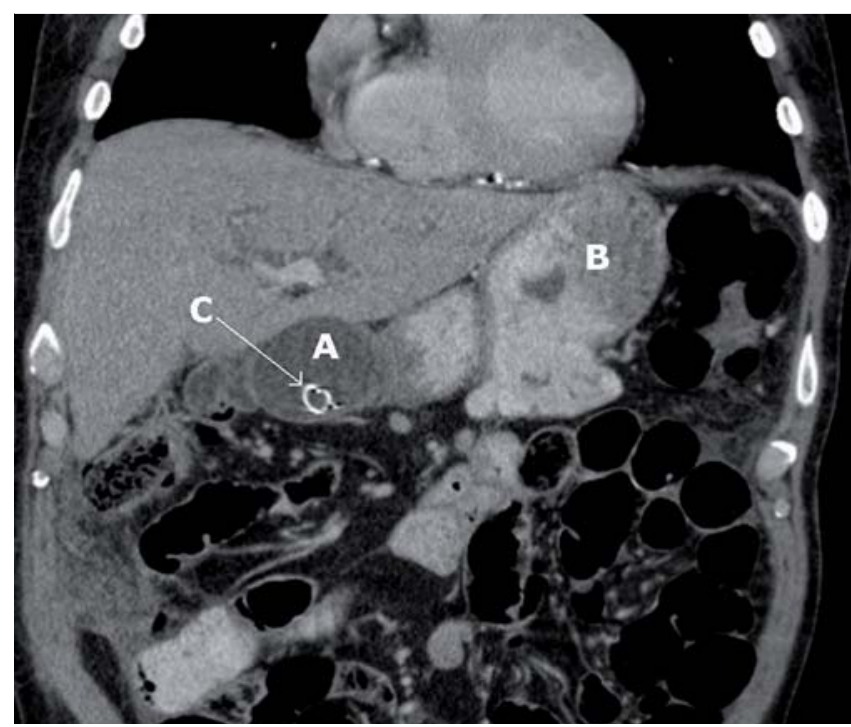

Fig. 1 Computed tomography scan reconstruction showing dilated hepaticojejunostomy loop (A), adjacent to the stomach (B), with biliary stent in situ (C).

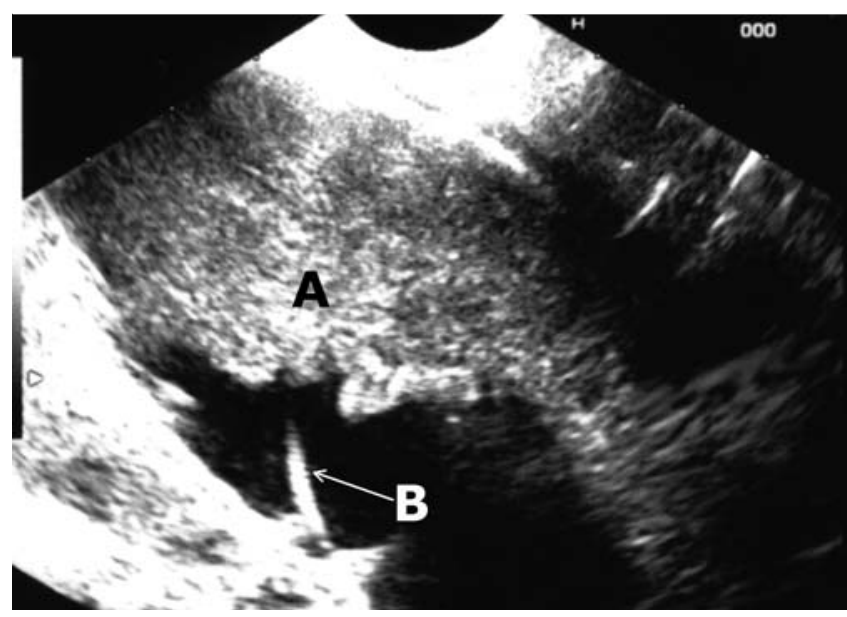

Fig. 2 Endoscopic ultrasound image of the dilated hepaticojejunostomy loop (A), with guide wire (B) in the collection.

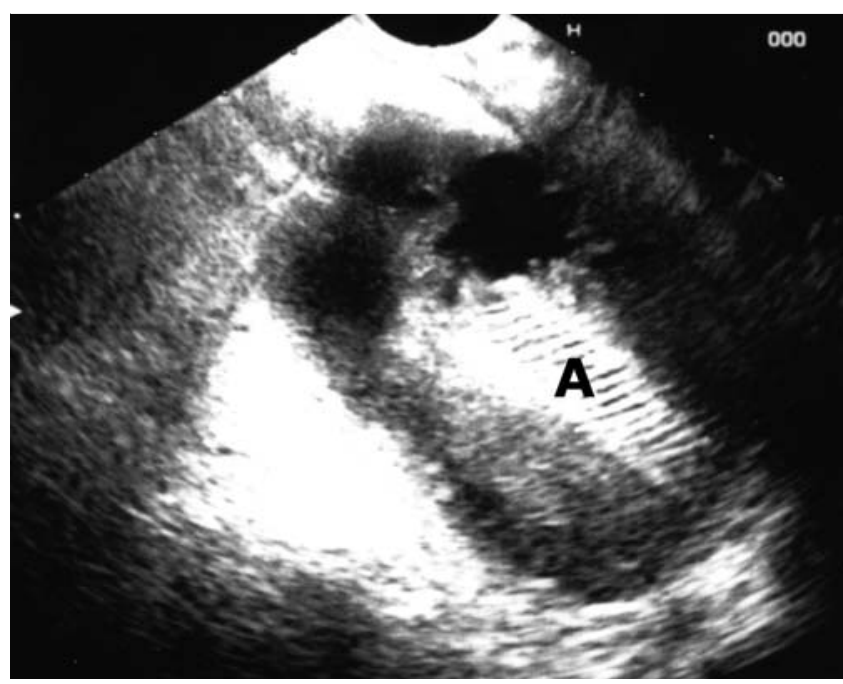

Fig. 3 Endoscopic ultrasound image of the metal stent $(A)$ in the collection after deployment. 


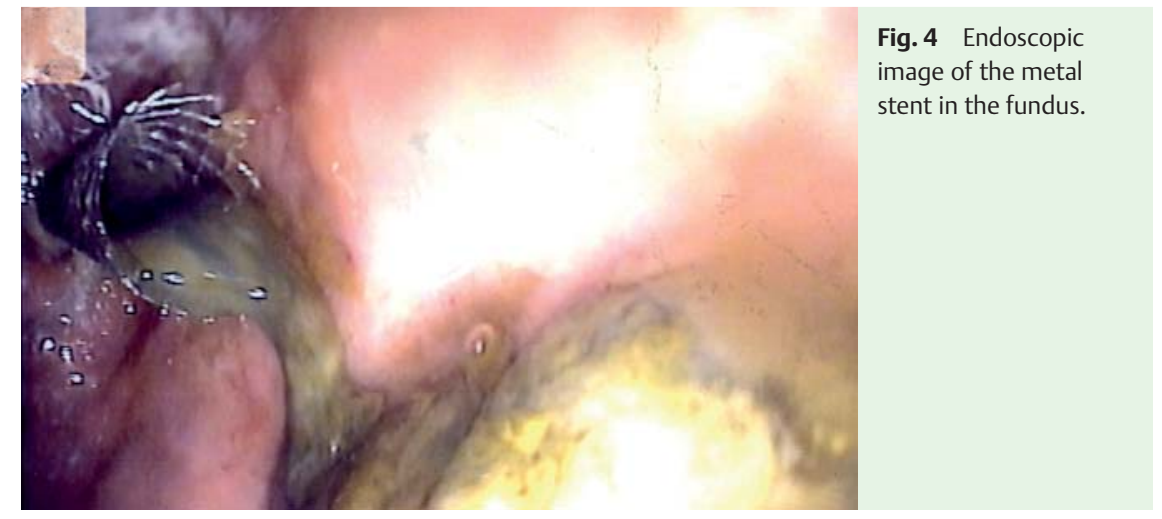

Endoscopy_UCTN_Code_TTT_1AS_2AG

Competing interests: None

S. Chatterjee ${ }^{1}$, B. Ibrahim ${ }^{1}$, R. M. Charnley ${ }^{2}$, J. Scott ${ }^{3}$, M. Nayar ${ }^{1}$

1 Department of Gastroenterology, Freeman Hospital, Newcastle-upon-Tyne, United Kingdom

2 Hepatic and Pancreato-Biliary Surgery, Freeman Hospital, Newcastle-upon-Tyne, United Kingdom

3 Department of Radiology, Freeman Hospital, Newcastle-upon-Tyne, United Kingdom
Bibliography

Dol 10.1055/s-0030-1255720

Endoscopy 2011; 43: E1 -E2

(c) Georg Thieme Verlag KG Stuttgart · New York . ISSN 0013-726X

\section{Corresponding author}

S. Chatterjee, MD. MRCP (UK)

Department of Gastroenterology Freeman Hospital

Newcastle-upon-Tyne NE7 7DN

United Kingdom

Fax: +44-191-2231249

suvadip_chatterjee@yahoo.com 